\title{
Influence of a Uniform Current on Collective Magnetization Dynamics in a Ferromagnetic Metal
}

\author{
J. Fernández-Rossier ${ }^{1,2}$, M. Braun ${ }^{1,3}$ A.S. Núñez ${ }^{1}$ and A. H. MacDonald ${ }^{1}$ \\ ${ }^{1}$ Physics Department, University of Texas at Austin, Austin, TX 78712. USA \\ 2 Departamento de Física Aplicada, Universidad de Alicante, \\ San Vicente del Raspeig 03690, Alicante, Spain and \\ ${ }^{3}$ Institut für Theoretische Festkörperphysik, Universität Karlsruhe, 76128 Karlsruhe, Germany
}

(Dated: February 2, 2008)

\begin{abstract}
We discuss the influence of a uniform current, $\vec{j}$, on the magnetization dynamics of a ferromagnetic metal. We find that the magnon energy $\epsilon(\vec{q})$ has a current-induced contribution proportional to $\vec{q} \cdot \overrightarrow{\mathcal{J}}$, where $\overrightarrow{\mathcal{J}}$ is the spin-current, and predict that collective dynamics will be more strongly damped at finite $\vec{j}$. We obtain similar results for models with and without local moment participation in the magnetic order. For transition metal ferromagnets, we estimate that the uniform magnetic state will be destabilized for $j \gtrsim 10^{9} \mathrm{~A} \mathrm{~cm}^{-2}$. We discuss the relationship of this effect to the spin-torque effects that alter magnetization dynamics in inhomogeneous magnetic systems.
\end{abstract}

PACS numbers:

\section{INTRODUCTION}

The strong and robust magnetotransport effects that occur in metallic ferromagnets (anisotropic, tunnel, and giant magnetoresistance for example [1]) result from the sensitivity of magnetization orientation to external fields, combined with the strong magnetization-orientation dependent potentials felt by the current-carrying quasiparticles. This fundamentally interesting class of effects has been exploited in information storage technology for some time, and new variations continue to be discovered and explored. Attention has turned more recently to a distinct class of phenomena in which the relationship between quasiparticle and collective properties is inverted, effects in which control of the quasiparticle state is used to manipulate collective properties rather than vice-versa. Of particular importance is the theoretical prediction [2, 3] of current induced magnetization switching and related spin transfer effects in ferromagnetic multilayers. The conditions necessary to achieve observable effects have been experimentally realized and the predictions of theory largely confirmed by a number of groups [4, 5, 6, 7, [8, 9] over the past several years.

Current-induced switching is expected [2, 3, 10] to occur in magnetically inhomogeneous systems containing two or more weakly coupled magnetic layers. The work presented in the present paper was motivated by a related theoretical prediction of Bazaily, Jones, and Zhang (hereafter BJZ), who argued that the energy functional of a uniform bulk half-metallic ferromagnet contains a term linear in the current of the quasiparticles 11, i.e. that collective magnetic properties can be influenced by currents even in a homogeneous bulk ferromagnetic metal. The current-induced term in the energy functional identified by BJZ implies an additional contribution to the Landau-Lifshitz equations of motion and, in a quantum theory, to a change proportional to $\vec{q} \cdot \vec{j}$ in the magnon energy $\epsilon(\vec{q})$. (Here $\vec{q}$ is the magnon or spin-wave wavevector and $\vec{j}$ is the current density in the ferromagnet.) The BJZ theory predicts that a sufficiently large current density will appreciably soften spin waves at finite wavevectors and eventually lead to an instability of a uniform ferromagnet. The current densities necessary to produce an instability were estimated by BJZ to be of order $10^{8} \mathrm{~A}$ $\mathrm{cm}^{-2}$, roughly the same scale as the current densities at which spin-transfer phenomena are realized [4, 5, 7, 8, 9], apparently suggesting to some that these two phenomena are deeply related.

In this paper we establish that modification of spinwave dynamics by current is a generic feature of all uniform bulk metallic ferromagnets, not restricted to the half-metallic case considered by BJZ. We find that, in the general case, the extra term in the spin wave spectrum

$$
\delta \epsilon(\vec{q}) \propto \vec{q} \cdot \overrightarrow{\mathcal{J}}
$$

where $\overrightarrow{\mathcal{J}}$ is the spin current, i.e., the current carried by the majority carriers minus the current carried by the minority carriers [12]. In the half metallic case $\overrightarrow{\mathcal{J}}=\vec{j}$, recovering the result of Reference 11. For reasons that will become apparent later, we refer to the extra term in the spin wave dispersion as the spin wave Doppler shift, although this terminology ignores the role of underlying lattice as we shall explain. We also study the effect of a uniform current on spin-wave damping. The usual Gilbert damping law $\gamma \propto \epsilon(\vec{q}=0)$, has an additional contribution proportional to the spin-current density. In our picture, a uniform current modifies collective magnetization dynamics because it alters the distribution of quasiparticles in momentum space.

Our paper is organized as follows. In Section II we present two general qualitative arguments which partially justify Eq.( (1), independent of any detailed microscopic model. In Section III we substantiate the arguments with 
a microscopic calculation of the spin wave spectrum for a ferromagnetic (but not necessarily half-metallic) phase of a Hubbard model, including the effect of the current. We derive Eq.(1), and demonstrate explicitly that when generalized from the half-metallic case to the general case, the spin wave Doppler shift is proportional to the spincurrent not the total current. The microscopic calculation of Section III uses an effective action approach, which separates collective and quasiparticle coordinates in a natural way and is well suited to study their interplay. In Section IV we specialize to the half-metallic case and re-derive the results of reference [11] for the case of an $s-d$ model ferromagnet. This serves the purpose of establishing a clear formal connection between the derivations presented in Sections II and III and the derivation presented by BJZ, which appear superficially to be quite distinct. In Section $\mathrm{V}$ we discuss the effect of a current on spin wave damping. We consider both damping due to the coupling of spin waves with the quasiparticles and two magnon damping, which we argue is enhanced by the spin wave Doppler shift of Eq.(1). In Section VI we discuss the relationship between the spin wave Doppler shift and spin-transfer in inhomogeneous ferromagnets. Finally, in Section VII we summarize our main results and present our conclusions.

\section{QUALITATIVE EXPLANATION OF THE CURRENT-INDUCED MAGNON ENERGY SHIFT}

The low energy collective dynamics of the magnetization orientation in a ferromagnet is described by the Landau-Lifshitz equation:

$$
\frac{\hbar d \vec{\Omega}(\vec{r}, t)}{d t}=\vec{\Omega} \times\left[\frac{\delta E\left(\vec{\Omega}, \partial_{i} \Omega_{j}\right)}{\delta \vec{\Omega}}+\alpha \hbar \frac{d \vec{\Omega}(\vec{r}, t)}{d t}\right]
$$

where $\vec{\Omega}(\vec{r}, t)$ is an unimodular vector field which describes the orientation of the collective magnetization and $E\left(\vec{\Omega}, \partial_{i} \Omega_{j}\right)$ is an energy functional of $\vec{\Omega}(\vec{r}, t)$ and its derivatives. The generic applicability of this equation follows from the collective nature of spin-dynamics in ferromagnets. It can be derived from a number of different microscopic models in a number of different ways. In particular, this equation describes the lowenergy long-wavelength dynamics of the two models of metallic ferromagnetism that we consider in later sections. Normally $E$ is minimized by a collinear configurations $\vec{\Omega}(\vec{r}, t)=\vec{\Omega}_{0}$ along some privileged easy direction. The Landau-Lifshitz equations linearized around $\vec{\Omega}_{0}$ have solutions which describe distortions of the magnetization orientation that propagate like waves with wave $\vec{q}$ and frequency $\omega(\vec{q})$ 13]. In a quantum treatment, magnetization orientation fluctuations are quantized in units of $\epsilon(\vec{q})=\hbar \omega(\vec{q})$

In a metallic ferromagnet, the quasiparticles occupy bands 14] that are energetically split by an effective Zeeman-coupling magnetic field oriented along the direction $\vec{\Omega}$. Non-collinear configurations are penalized because band-electron kinetic energies are raised by an inhomogeneous effective field $\vec{\Omega}(\vec{r}, t)$. The easy axis is determined by spin-orbit interactions of the band electrons and by the magnetostatic energy, which because of its long range depends on the overall shape of the sample.

The dynamics generated by the first term in square brackets in Eq. (2) is energy conserving whereas the second term, proportional to the dimensionless coefficient $\alpha$, transfers energy from the collective coordinate to other degrees of freedom. In a metallic ferromagnet, the damping is partly due to the excitation of electron-hole pairs in response to the temporal evolution of $\vec{\Omega}$. It is clear, therefore, that there is an intimate relation between the dynamics of the collective coordinate and the state of the quasiparticles. What's more, when current flows inside a ferromagnet, the momentum-space distribution functions that describe quasiparticle state occupation probabilities are altered. It is natural, therefore, to expect that the dissipative dynamics of the collective magnetization will be affected by current flow. In Ref. 11 it was shown that, in a half metallic ferromagnet modeled by a s-d model (a model with a single band coupled by exchange interactions to local moments), the energy functional $E$ has a term linearly proportional to the quasiparticle current, $\vec{j}$. In the following paragraphs we present three arguments to support the idea that the spin wave spectrum of any metallic ferromagnet is modified by a uniform current in a manner similar to that suggested by Eq.(1)

We start with the simplest case, a half-metallic ferromagnetic electron gas, in which the current effect can be understood simply in terms of Galilean invariance. The current carrying state of this system is simply one in which the entire electronic systems moves along with a drift velocity $\vec{v}_{D}$. A spin wave excitation is one in which the magnetization orientation precesses around the easy axis with frequency $\omega(\vec{q})$ :

$$
\hat{\Omega}=\left(\epsilon \sin \left(\vec{q} \cdot \vec{r}-\omega_{0}(\vec{q}) t\right), \epsilon \cos \left(\vec{q} \cdot \vec{r}-\omega_{0}(\vec{q}) t\right), 1-\epsilon / 2\right)
$$

In the lab frame, the system is seen as moving with velocity $\vec{v}_{D}$, and carrying current $\vec{j}=-n e \vec{v}_{D}$. The fixed position $\vec{r}_{L}$ in the lab frame, has position $\vec{r}_{L}-\vec{v}_{D} t$ in the moving frame. The precession frequency seen at a fixed lab frame position is therefore Doppler shifted to $\omega_{0}(\vec{q})+\vec{q} \cdot \vec{v}_{D}$.

This simple effect is the essence of the spin-wave Doppler shift. In terms of the current density the spinwave Doppler shift in the magnon energy is $\hbar \vec{q} \cdot \vec{j} / e n$. Systems of practical interest are neither Galilean invariant nor, with a few possible exceptions, half metallic, however so a more detailed analysis is required to deter- 
mine how the spin-wave Doppler shift is manifested in real systems.

A second useful point of view follows from considering a single-mode-approximation for the quantum spin-wave energy $\epsilon(\vec{q})=\hbar \omega(\vec{q})$. Elementary magnon excitations of a ferromagnet reduce the total spin projection along the easy axis by one unit and add crystal momentum $\hbar \vec{q}$. A state with the correct quantum numbers can be generated starting from the ferromagnetic ground state (or from a state that carries a uniform current) $\left|\Psi_{0}\right\rangle$ by acting on it with the 'magnon creation operator'

$$
s_{-}(-\vec{q})=\sum_{i=1, N} s_{-i} \exp (i \vec{q} \cdot \vec{r})
$$

where $s_{-i}$ is the spin-lowering operator for the $i$-th particle. Two-particle Greens functions constructed from this operator have poles with large residues at magnon excitation energies. The single-mode approximation consists of using $|\Psi(\vec{q})\rangle \equiv s_{-}(-\vec{q})\left|\Psi_{0}\right\rangle$ as a variational wavefunction for the magnon state at wavevector $\vec{q}$. Given this approximation for the magnon state, its excitation energy

$$
\epsilon(\vec{q}) \equiv \frac{\langle\Psi(\vec{q})|\mathcal{H}| \Psi(\vec{q})\rangle}{\langle\Psi(\vec{q}) \mid \Psi(\vec{q})\rangle}-E_{0}
$$

can be expressed in terms of the expectation value of a commutator between the general many-particle Hamiltonian $\mathcal{H}$ and either magnon creation or annihilation operators and simplified to the following form:

$$
\begin{aligned}
& \epsilon(\vec{q})=\frac{\hbar^{2} q^{2}}{2 m}+ \\
& \frac{\hbar \vec{q}}{m} \cdot \frac{\sum_{i j}\left\langle\Psi_{0}\left|s_{+i} s_{-j} \exp \left[i \vec{q} \cdot\left(\vec{r}_{j}-\vec{r}_{i}\right)\right] \vec{p}_{j}\right| \Psi_{0}\right\rangle}{\left\langle\Psi_{0}\left|s_{+}(\vec{q}) s_{-}(\overrightarrow{-q})\right| \Psi_{0}\right\rangle}
\end{aligned}
$$

The second term on the right hand side of Eq.( 6 ) is the magnon Doppler shift term. In this term $s_{ \pm i}$ and $\vec{p}_{i}$ are the spin raising and lowering and momentum operators for particle $i$. The numerator and denominator of this term are, in general, complex two-particle correlation functions. The correlation functions are simplified when the ferromagnetic state is approximated by a Slater determinant with definite occupation numbers for both majority $(\uparrow)$ and minority $(\downarrow)$ spin momentum states, i.e. by the electron gas Stoner model ferromagnetic ground state. Then to leading order in $\vec{q}$ we find that the magnon Doppler shift has the value

$$
\delta \epsilon(\vec{q})=\frac{\hbar \vec{q}}{m} \cdot \frac{\overrightarrow{\mathcal{J}}}{n_{\uparrow}-n_{\downarrow}} .
$$

Eq. 7 is most easily obtained by writing the operators whose expectation values need to be evaluated as a sum of one-body and two-body terms and then using standard second quantization identities. The most important conclusion suggested by this equation is that, at least for parabolic bands, in generalizing the magnon Doppler effect from half-metallic ferromagnets to ferromagnets with states of both spins occupied, the current is replaced by the spin-current $\overrightarrow{\mathcal{J}}$, and the density by the spin-density.

Finally, the same result can be derived by considering a variational wave function for the spin-wave state of a ferromagnetic metal in which all quasiparticle states that are singly occupied share a common spinor that describes long-wavelength spatial precession around the easy direction. For example if the $\hat{x}$ direction is the easy direction the spinor that describes small amplitude precession is $(u, v)=\left(1-\eta^{2} / 2, \eta \exp (i \vec{q} \cdot \vec{r})\right)$. The $\vec{q} \cdot \overrightarrow{\mathcal{J}}$ correction then follows by observing that the magnon energy equals the energy change divided by the change in the $\hat{x}$ direction magnetization component, with both quantities being proportional to $\eta^{2}$ at small $\eta$. This findings suggest that the explicit approximate expression for the magnon Doppler shift, derived from the SMA for parabolic bands, is likely to qualitatively correct even for realistic ferromagnets with more complicated band structures. Indeed, that is the conclusion that follows from the more microscopic derivations in the following two sections.

\section{CURRENT DRIVEN SPIN WAVES IN A HUBBARD MODEL FERROMAGNET}

In order to explain our theory of the influence of uniform currents on the spin-wave spectrum, we first recall how spin-waves and quasiparticle states are related in equilibrium. This development will also establish the notation we use for the non-equilibrium case. The description we use is one in which a collective fluctuation field interacts with fermionic quasiparticle fields. It allows us to borrow from standard theories of quantum harmonic oscillators weakly coupled to a bath, in order to generalize the theory of collective dynamics from equilibrium to non-equilibrium cases.

\section{Hamiltonian and effective action}

In the previous section we discussed three general arguments in support of the existence of a spin wave Doppler shift in a metallic ferromagnet that is proportional to the spin current as in Eq.(1). We now look more closely at the underlying physics by carrying out an explicit microscopic calculation of the spin waves for a Hubbard model in the presence of a current. Unlike the s-d model considered in Ref. 11, the Hubbard model allows for ferromagnetism in a system with only itinerant electrons. The Hubbard model Hamiltonian is [16] :

$$
\mathcal{H}=\sum_{i, j} t_{i j} c_{i, \sigma}^{\dagger} c_{j, \sigma}+U \sum_{j} n_{j, \uparrow} n_{j, \downarrow}
$$

The elementary excitations of a metallic ferromagnet 
are quasiparticles and spin waves. We want to derive the propagator for the spin waves of the ferromagnetic phase of this model and to see how is affected by a quasiparticle current. To do so, it is convenient to use the functional integration approach, [15, 16, 17, 18], in which the quasiparticles are integrated out and an effective action for the spin waves is obtained. This procedure is sketched below, the details can be found in Refs. 15, 16, 17, 18, 19. The final result for the spin wave spectrum is equivalent to that obtained by doing a Random Phase Approximation (RPA) 20] calculation. However, the effective action approach provides a convenient conceptual framework to understand the connection between spin waves and nonequilibrium quasiparticle states, the central focus of this paper.

The interaction term in the Hubbard model can be written as 16]

$$
U \sum_{j} n_{j, \uparrow} n_{j, \downarrow}=-\frac{2}{3} U \sum_{i} \vec{S}_{i}^{2}+\frac{U}{2} \sum_{\sigma, i} n_{\sigma, i}
$$

We represent the partition function of this model as a path integral over fermion coherent states [22], labeled by $\left\{\bar{\Psi}_{\alpha}, \Psi_{\alpha}\right\}$, where $\alpha \equiv i, \sigma$. The key idea which allows quasiparticle and collective degrees of freedom to be separated, while still treating the magnetization as a quantum field, is the introduction of a Hubbard-Stratonovich transformation 21] to represent the interaction term. By making this transformation, we trade a problem of interacting fermions for a problem of independent fermions whose spin is coupled to a bosonic spin-splitting effective magnetic field $\vec{\Delta}_{i}(\tau)$, which acts as the collective magnetic coordinate. The partition function reads:

$\mathcal{Z}=\int \mathcal{D} \bar{\Psi}_{\alpha}(\tau) \mathcal{D} \Psi_{\alpha}(\tau) \mathcal{D} \vec{\Delta}_{i}(\tau) \exp \left[-S\left(\bar{\Psi}_{\alpha}, \Psi_{\alpha}, \vec{\Delta}_{i}\right)\right]$

where the action is

$$
S=\int_{0}^{\beta} d \tau \sum_{i} \frac{3 \vec{\Delta}_{i}(\tau)^{2}}{8 U}+\sum_{i, i^{\prime}, \sigma} \bar{\Psi}_{i, \sigma}(\tau) \mathcal{G}_{i j, \sigma, \sigma^{\prime}}^{-1} \Psi_{j, \sigma}(\tau)(9)
$$

and

$$
\mathcal{G}_{i j, \sigma, \sigma^{\prime}}^{-1}=\left(\partial_{\tau}-\mu+\frac{U}{2}\right) \delta_{i, j}+t_{i, j}-\vec{\Delta}_{i} \cdot \frac{\vec{\tau}_{\sigma, \sigma^{\prime}}}{2} \delta_{i, j}
$$

is the inverse of the Green's function operator.

The action (9) is the sum of three terms, i) noninteracting tight binding fermions (with a Hartree shift), ii) a term quadratic in the bosonic field and iii) their coupling $\vec{\Delta}_{i} \cdot \vec{S}_{i}$, where $\vec{S}_{i}(\tau) \equiv \sum_{\sigma, \sigma^{\prime}} \frac{1}{2} \bar{\Psi}_{i, \sigma} \vec{\tau}_{\sigma, \sigma^{\prime}} \Psi_{i^{\prime}, \sigma^{\prime}}$. Since the action is quadratic in the fermion variables, the fermion functional integral can be formally evaluated. This allows to write the partition function as a path integral over the auxiliary field $\vec{\Delta}$ only,

$$
\mathcal{Z}=\int \mathcal{D} \vec{\Delta}_{i}(\tau) e^{-S_{\text {eff }}(\vec{\Delta})}
$$

where the effective action reads:

$$
S_{\mathrm{eff}}(\vec{\Delta})=\int_{0}^{\beta} d \tau \sum_{i} \frac{3 \vec{\Delta}_{i}(\tau)^{2}}{8 U}-\operatorname{Tr} \operatorname{Ln}\left[\mathcal{G}^{-1}(\vec{\Delta})\right]
$$

Eqs.(11) and (12) are one of the many possible representations of the exact partition function for the Hubbard Model. The effective action (12) describes a complicated quantum field theory for $\vec{\Delta}_{i}(\tau)$.

\section{Mean Field theory: Spin-split bands}

The first step in a field theory of ferromagnetism is usually to look for classical solutions, i.e. for field configuration $\vec{\Delta}_{i}^{\mathrm{cl}}(\tau)$ for which the effective action is stationary. The saddle point equation reads $\vec{\Delta}_{i}^{\mathrm{cl}}=\frac{4 U}{3}\left\langle\vec{S}_{i}\right\rangle$, where the average is computed with a Green function $\mathcal{G}\left(\vec{\Delta}^{\mathrm{cl}}\right)$ obtained by replacing, in Eq.(10), the fluctuating field $\vec{\Delta}_{i}(\tau)$ by the saddle point solution.

Assuming the existence of a ferromagnetic mean-field state, the classical solution for a perfect crystal is static (independent of $\tau$ ) and homogeneous (independent of $i$ ). It is therefore characterized by a direction $\mathbf{n}$ and a length $\left|\vec{\Delta}^{\mathrm{cl}}\right| \equiv \Delta$. Because of the spin rotational invariance of the Hubbard Hamiltonian, $\mathbf{n}$ is arbitrary. In real systems $\mathbf{n}$ is determined by spin-orbit interactions and magnetostatic effects. The mean-field Green's function, $\mathcal{G}\left(\vec{\Delta}^{\mathrm{cl}}\right)$, describes fermions which occupy bands that are spin-split by an effective magnetic field along $\mathbf{n}$ (See Fig.(1)). The magnitude of the spin splitting, $\Delta$, is obtained from the saddle point equations, which, for this simple model, reduce to the following form:

$$
\Delta=\frac{4 U}{3} \frac{1}{2 \mathcal{N}} \sum_{\vec{k}}\left[n_{F}\left[\epsilon_{\vec{k}}^{\uparrow}\right]-n_{F}\left[\epsilon_{\vec{k}}^{\downarrow}\right]\right]
$$

where $\epsilon_{\vec{k}}^{\sigma}=\epsilon(\vec{k})-\sigma \frac{\Delta}{2}$ are the quasiparticle energies of the spin-split bands and $\mathcal{N}$ is the number of lattice sites. Notice that the majority band has spins parallel to $\mathbf{n}$, denoted by $\uparrow$. The saddle point equations show explicitly that the auxiliary field $\vec{\Delta}^{\mathrm{cl}}$ is proportional to the average fermion magnetization, which usually appears as the fundamental field in classical micromagnetic theories for realistic magnetic materials. Hereafter we refer to $\vec{\Delta}(\tau)$ as the

\section{Spin waves without current}

We are interested in the dynamics of the collective coordinate, so that the static solution obtained by solving the mean field approximation is insufficient. To describe the elementary collective excitations we need to study small amplitude dynamic fluctuations of the collective 


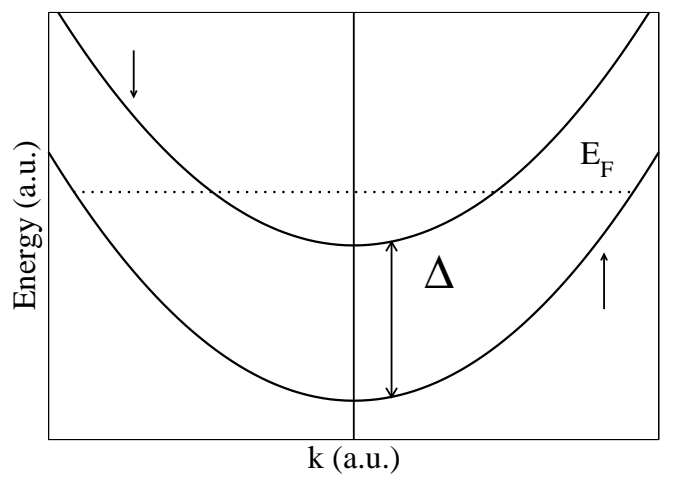

FIG. 1: Mean field quasiparticle bands. Dashed line shows the Fermi Energy. $\Delta$ is the spin splitting energy.

coordinate around the static solution:

$$
\vec{\Delta}_{i}(\tau) \simeq \vec{\Delta}^{\mathrm{cl}}+\delta \vec{\Delta}_{i}(\tau)
$$

We introduce Eq.(14) into the effective action (Eq.(12) and neglect terms of order $\left[\delta \vec{\Delta}_{i}(\tau)\right]^{3}$ and higher. The resulting action $S_{\mathrm{cl}}\left(\vec{\Delta}^{\mathrm{cl}}\right)+S_{\mathrm{SW}}$, where the first term is the classical approximation to the effective action and the fluctuation correction is:

$$
S_{\mathrm{SW}}=\frac{1}{2 \beta \mathcal{N}} \sum_{\mathcal{Q}, a, b} \delta \Delta_{a}(\mathcal{Q}) \mathcal{K}_{a b}(\mathcal{Q}) \delta \Delta_{b}(-\mathcal{Q})
$$

where $\mathcal{Q}$ is a shorthand for $\vec{q}, i \nu_{n}$, and $a, b$ stand for Cartesian coordinates. Note that the bosonic fields, $\delta \vec{\Delta}(\mathcal{Q})$ are dimensionless and the Kernel $\mathcal{K}$ has dimensions of inverse energy. This action defines a field theory for the spin fluctuations. The equilibrium Matsubara Green function, $\mathcal{D}_{a b}\left(\vec{q}, i \nu_{n}\right)$, is given 22, 23] by the inverse of spin fluctuation Kernel, $\mathcal{K}_{a b}(\mathcal{Q})$. Analytical expressions for $\mathcal{K}_{a b}(\mathcal{Q})$ are readily evaluated for the case of parabolic bands and are appealed to below. We obtain the retarded spin fluctuation propagator by analytical continuation of the Matsubara propagator: $D_{a b}^{\mathrm{ret}}(\vec{q}, \omega)=\mathcal{D}_{a b}\left(\vec{q}, i \nu_{n} \rightarrow \omega+i 0^{+}\right)$ The imaginary part of the retarded propagator summarizes the spectrum and the damping of the spin fluctuations most directly.

The theory defined by Eq. (15) includes two types of spin fluctuations which are very different: i) longitudinal fluctuations (parallel to $\mathbf{n}$ ), or amplitude modes and ii) transverse fluctuations (perpendicular to $\mathbf{n}$ ), or spin waves. The amplitude modes involve a change in the magnitude of the local spin splitting, $\Delta$, and are either over damped or appear at energies above the continuum of spin-diagonal particle-hole excitations. In contrast, the spin waves are gapless in the limit $\vec{q}=0$, in agreement with the Goldstone theorem, and are often weakly damped even in realistic situations. Note that the amplitude modes decouple from the spin wave modes for small amplitude fluctuations. For $\hat{x}=\mathbf{n}$, we can write

$$
\mathcal{K}_{a b}(\mathcal{Q})=\left[\begin{array}{ccc}
\mathcal{K}^{\prime \prime} & 0 & 0 \\
0 & \mathcal{K}_{y y} & \mathcal{K}_{y z} \\
0 & \mathcal{K}_{z y} & \mathcal{K}_{z z}
\end{array}\right]
$$

Since the low energy dynamics of a metallic ferromagnet is governed by transverse spin fluctuations, we do not discuss longitudinal fluctuations further. After analytic continuation, we obtain the following result for the inverse of the retarded transverse spin fluctuation Green function $\left(D^{\text {ret }}\right)^{-1}$, which is diagonal when we rotate from $\hat{y}, \hat{z}$ to $+\hat{z} \pm i \hat{y}$ chiral representations. The diagonal elements are then

$$
D_{ \pm}^{\mathrm{ret}}(\vec{q}, \omega)=\frac{4 U}{3} \frac{1}{1+\frac{2}{3} U \Gamma( \pm \vec{q}, \pm \omega)}
$$

where $\Gamma(\vec{q}, \omega)$ is the Lindhard function evaluated with the spin-split mean-field bands:

$$
\Gamma(\vec{q}, \omega)=\frac{1}{\mathcal{N}} \sum_{\vec{k}} \frac{n_{\vec{k}}^{\uparrow}-n_{\vec{k}+\vec{q}}^{\downarrow}}{\epsilon_{\vec{k}}^{\uparrow}-\epsilon_{\vec{k}+\vec{q}}^{\downarrow}+\omega+i 0^{+}}
$$

where $n_{\vec{k}}^{\sigma}$ is shorthand for the Fermi-Dirac occupation function $n_{F}\left[\epsilon_{\vec{k}}^{\sigma}\right]$ for the quasiparticle occupation numbers. Eqs. (17) and (18) make it clear that the spin wave spectrum is a functional of the occupation function $n_{F}$ for the quasi-particles in the spin-split bands. The influence of a current on the spin-wave spectrum will enter our theory through non-equilibrium values of these occupation numbers.

In the case of parabolic bands (still without current), the Taylor expansion of the Lindhardt function in the low-energy low-frequency limit gives the following result for the spin wave propagator:

$$
D_{ \pm}^{\mathrm{ret}}(\vec{q}, \omega)=\frac{4 U \Delta}{3} \frac{1}{\omega \pm \rho q^{2}}
$$

where $\rho$ is the spin stiffness which is easily computed analytically for the case of parabolic bands. The poles of Eq.(19) give the well known result for the spin wave dispersion, $\omega= \pm \rho q^{2}$. Several remarks are in order: i) In real systems, spin-orbit interactions lift spin rotational invariance, resulting in a gap for the $q=0$ spin waves. The size of the gap is typically of order of $1 \mu \mathrm{eV}$ [24]. ii) The interplay between disorder and spin orbit interactions, absent in the above model, gives rise to a broadening of the spin wave spectrum, even at small frequency and momentum. In Section $\mathrm{V}$ we address this issue and discuss how damping is changed in the presence of a current.

\section{Spin waves with current}

In the previous subsection we derived the spin wave spectrum of a metallic ferromagnet in thermal equilib- 
rium. Equations (17) and (18) establish a clear connection between spin waves and quasiparticle distributions. In order to address the same problem in the presence of a current, a non-equilibrium formalism is needed. By taking advantage of the formulation discussed above in which collective excitations interact with fermion particle-hole excitations we are able to appeal to established results for harmonic oscillators weakly coupled to a bath. In the equilibrium case, the fact that the lowenergy Hamiltonian for magnetization-orientation fluctuations is that of a harmonic oscillator follows by expanding the fluctuation action to leading order in $\omega$ to show that $\hat{y}$ and $\hat{z}$ direction fluctuations are canonically conjugate. In our model magnons are coupled to a bath of spin-flip particle-hole excitations. Following systembath weak coupling master equation analyses 25] we find that the collective dynamics in the presence of a nonequilibrium current-carrying quasiparticle system differs from the equilibrium one simply by replacing Fermi occupation numbers by the non-equilibrium occupation numbers of the current-carrying state. The following term therefore appears in the Taylor expansion of the Lindhardt function $\Gamma$ :

$$
\left.\frac{\partial \Gamma}{\partial q_{i}}\right|_{q=\omega=0}=\frac{1}{\mathcal{N} \Delta^{2}} \sum_{\vec{k}} \frac{\partial \epsilon(\vec{k})}{\partial k_{i}}\left[n_{\vec{k}}^{\uparrow}-n_{\vec{k}}^{\downarrow}\right]
$$

Since this expression uses the easy direction $\hat{x}$ as the spinquantization axis, the $x$ (spin) component of the spin current is:

$$
\overrightarrow{\mathcal{J}} \equiv \frac{e}{\hbar \mathcal{N}} \sum_{\vec{k}} \frac{\partial \epsilon(\vec{k})}{\partial \vec{k}}\left[n_{\vec{k}}^{\uparrow}-n_{\vec{k}}^{\downarrow}\right]
$$

so that

$$
\left.\frac{\partial \Gamma}{\partial q_{i}}\right|_{q=\omega=0}=\frac{\hbar}{e \Delta^{2}} \mathcal{J}_{i}
$$

The quantity $\mathcal{J}_{i}$, the component of the spin current polarized along the magnetization direction $\mathbf{n}=\hat{x}$ and flowing along the $i$ axis, is the difference between the current carried by majority and minority carriers. In equilibrium there is no current and no linear term occurs in the wavevector Taylor series expansion, leading to quadratic magnon dispersion as obtained in Eq.(19). When (charge) current flows through the ferromagnet, the difference in carrier density and mobility between majority and minority bands inevitably gives rise to a nonzero spin current [30]. We therefore obtain the following spectrum for spin waves in the presence of a current:

$$
\omega=\rho q^{2}-\frac{2 U}{3 \Delta} \frac{\hbar}{e} \vec{q} \cdot \overrightarrow{\mathcal{J}}
$$

This equation is the central result of our paper. Notice that it is in precise agreement with the single-modeapproximation expression since $\Delta=\frac{2 U}{3}\left(n_{\uparrow}-n_{\downarrow}\right)$; in that case, however, the explicit expression was derived for the case of free-particle parabolic bands only. Eq.( 23) states that the spin wave spectrum of metallic ferromagnet driven by a current is modified in proportion to the resulting spin current.

In the half metallic case, when the density of minority carriers is zero, the spin current is equal to the total current and we recover the result of BJZ [11]. In that limit $\Delta=\frac{2 U}{3} n$ and $\rho \simeq \frac{\hbar^{2}}{2 m}$, leading to

$$
\omega=\frac{\hbar^{2}}{2 m} q^{2}-\frac{\hbar}{e n} \vec{q} \cdot \vec{j}=\frac{\hbar^{2}}{2 m} q^{2}-\hbar \vec{q} \cdot \vec{v}_{D}
$$

where we have expressed the current as $\vec{j}=e n \vec{v}_{D}$ with $\vec{v}_{D}$ the drift velocity, generalizing the half-metallic simple Doppler shift result to non-parabolic bands.

\section{Spin wave instability}

Eqs. (23) and (24), taken at face value, predict that the energy of a spin waves is negative and therefore that the uniform ferromagnetic state is destabilized by an arbitrarily small current. If this were really true, it would presumably be a rather obvious and well known experimental fact. It is not true because spin waves in real ferromagnetic materials have a gap due to both spin-orbit interactions and magnetostatic energy. Inserting by hand this (ferromagnetic resonance) gap, the spin wave dispersion reads:

$$
\omega=\omega_{0}+\rho q^{2}-\frac{2 U}{3 \Delta} \frac{\hbar}{e} \vec{q} \cdot \overrightarrow{\mathcal{J}}
$$

so that it takes a critical spin current to close the spin wave gap. In Fig.(2) we plot the current driven spin wave spectrum assuming $\omega_{0}=1 \mu \mathrm{eV}$, the electronic density of iron $\left(n=1.1710^{23} \mathrm{~cm}^{-3}\right)$ and a Doppler shift given by $q v_{D}$. The critical current so estimated is $\sim 1.110^{9} \mathrm{~A}$ $\mathrm{cm}^{-2}$ for a typical system. This critical current could be much lower, perhaps by several orders of magnitude, in metallic ferromagnets in which material parameters have been tuned to minimize the spin-wave gap. Experimental searches for current-driven anomalies in permalloy thin films, for example, could prove to be fruitful.

\section{Spin wave action with current}

In the small $\omega$ and small $\vec{q}$ limit, the spin waves are independent and their action is equivalent to that of an ensemble of non interacting harmonic oscillators,indexed with the label $\vec{q}$. The Matsubara action for a single oscillator mode is the frequency sum of

$$
\left[p_{\vec{q}}, x_{\vec{q}}\right]\left(\begin{array}{cc}
\frac{1}{2 M_{\vec{q}}} & -i \frac{\omega}{2} \\
i \frac{\omega}{2} & \frac{K_{\vec{q}}}{2}
\end{array}\right)\left[\begin{array}{c}
p_{\vec{q}} \\
x_{\vec{q}}
\end{array}\right]
$$




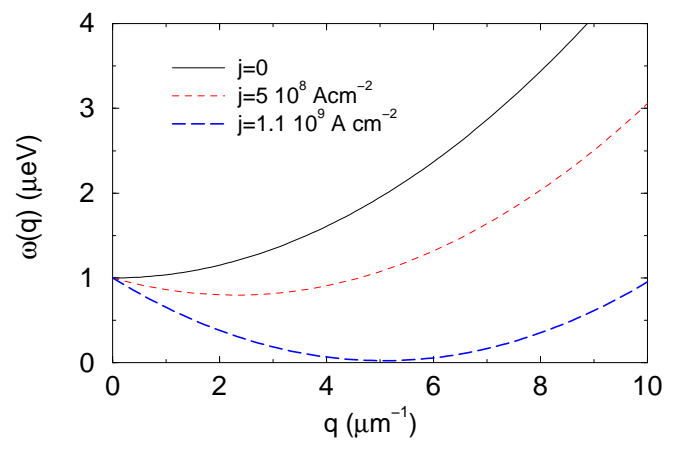

FIG. 2: Current modified spin-wave spectrum

where the diagonal terms are the Hamiltonian part of the action and the off-diagonal term can be interpreted as a Berry phase. For the spin waves, the analog of $p$ and $x$ are, modulo some constants, $\delta \Delta_{y}, \delta \Delta_{z}$. In this representation, the low $\omega$ and low $\vec{q}$ spin wave action reads:

$$
\chi_{\perp}^{-1}(\omega, \vec{q})=\left(\begin{array}{cc}
\rho \vec{q} \cdot \vec{q} & -i \omega \\
i \omega & \rho \vec{q} \cdot \vec{q}
\end{array}\right)+\frac{2 U}{3 \Delta} \frac{\hbar}{e} \overrightarrow{\mathcal{J}} \cdot \vec{q}\left(\begin{array}{cc}
0 & -i \\
i & 0
\end{array}\right)(27)
$$

This representation makes it clear that the spin wave Doppler shift appears as a modification of the term which couples the canonically conjugate variables, $\delta \Delta_{y}$ and $\delta \Delta_{z}$, i.e., the spin wave Doppler shift modifies the Berry phase. When expressed in this way, the spin-wave Doppler shift is partly analogous to the change in superfluid velocity in a superfluid that carries a finite mass current, and the stability limit we have discussed is partly analogous to the Landau criterion for the critical velocity of a superfluid. These analogies are closer in the case of ideal easy-plane ferromagnets, which like superfluids have collective modes with linear dispersion instead of a having a gap.

\section{ALTERNATE DERIVATION OF SPIN-WAVE DOPPLER SHIFT}

In the previous section we have used a functional integral approach to calculate how the spin wave propagator of a Hubbard model metallic ferromagnet is modified when current flows through the system. The BJZ derivation of the same effect was based on an identity at the operator level. BJZ used a $s-d$ model, i.e. a Hamiltonian for itinerant $(s)$ electrons interacting with localized (d) spins via an exchange interaction. They considered the limit of very large exchange interaction and low $s$ electron density, so that, in the ground state, the electrons are fully spin polarized. They then introduced a local spin rotation transformation defined so that at every point of the space the spin of the $s$ electrons is parallel with the local value of the $d$ electrons magnetic moment. This unitary transformation has been previously used for both $s-d$ and other microscopic models of ferromagnetism [18, 26, 27, 28, 29]. In the transformed frame, the exchange interaction is always diagonal in the spin index but the expression for the kinetic energy is complicated, and includes new terms. One of the new terms couples the $s$ electron current to a space derivative of the local spin magnetization. It is from this term in the exchange energy that BJZ derived the modification of the LandauLifshitz equations that we have identified as a spin-wave Doppler shift. In this section we bridge the gap between the two derivations. We recover the half metallic s-d Hamiltonian result of BJZ in a systematic way.

The continuum s-d model describes itinerant electrons, $\psi_{\sigma}$, interacting with a continuum of localized quantum spins, $\vec{M}(\vec{x})$, through a exchange interaction of strength $J$. The Hamiltonian for parabolic bands is given by:

$$
\mathcal{H}=\int d \vec{x} \sum_{\sigma, \sigma^{\prime}} \psi_{\sigma}^{\dagger}\left(-\frac{\hbar^{2} \vec{\nabla}^{2}}{2 m} \delta_{\sigma, \sigma^{\prime}}-\frac{J \vec{\tau}_{\sigma, \sigma^{\prime}}}{2} \cdot \vec{M}(\vec{x})\right) \psi_{\sigma^{\prime}}
$$

where $\vec{\tau}$ are the Pauli matrices. In order to derive an effective theory for the collective behavior of this system, we express its partition function as a coherent state path integral:

$$
\mathcal{Z}=\int \mathcal{D}^{2} \Psi_{\sigma}(\vec{x}, \tau) \mathcal{D} \vec{\Omega}(\tau) e^{-S_{B}+\int_{0}^{\beta} d \tau \bar{\Psi}_{\sigma^{\prime}}\left(\partial_{\tau}-\mu\right) \Psi_{\sigma^{\prime}}-\mathcal{H}}
$$

where $\tau$ is imaginary time, $\vec{\Omega}(\vec{x}, \tau)=\frac{1}{S} \vec{M}(\vec{x}, \tau)=$ $[\cos (\phi) \sin (\theta), \sin (\phi) \sin (\theta), \cos (\theta)]$ is the unimodular vector field which labels the spin coherent states, $S_{B}$ is the Berry phase term that captures the spin commutation relations [23], and $\Psi$ are the Grassmann numbers which label the fermion coherent states 22].

Following BJZ, we perform a unitary transformation on the spins of the itinerant electrons so that, at each point of time and space, the quantization axis is parallel to $\vec{M}(\vec{x}, \tau)$. BJZ considered only the limit of very strong ferromagnetic $J$, so that the spins of the occupied electronic states are always parallel to $\vec{M}(\vec{x}, \tau)$ and we can ignore the antiparallel electrons. This approximation is valid in half-metallic systems for energies much smaller than $J$, the local spin splitting. In this approximation the action for the parallel fermions in the rotated frame, denoted by $\Phi(\vec{x}, \tau)$, can then be written as $S=S_{B}+S_{0}+S_{1}+S_{2}$ where:

$$
\begin{aligned}
S_{B} & =\int_{0}^{\beta} d \tau \int d \vec{x} i S c_{S}\left(1+\frac{\bar{\Phi} \Phi}{2 S c_{S}}\right) \cos (\theta) \partial_{\tau} \phi \\
S_{0} & =\int_{0}^{\beta} d \tau \int d \vec{x} \bar{\Phi}\left(\partial_{\tau}-\mu-\frac{\hbar^{2} \nabla^{2}}{2 m}-J S\right) \Phi \\
S_{1} & =\frac{\hbar^{2}}{2 m} \int_{0}^{\beta} d \tau \int d \vec{x}\left(\sum_{i, j} \nabla_{i} \Omega_{j} \nabla_{i} \Omega_{j}\right) \frac{1}{4} \bar{\Phi} \Phi \\
S_{2} & =\frac{2}{e c} \int_{0}^{\beta} d \tau \int d \vec{x}\left[\overrightarrow{\mathcal{J}}_{P}+\overrightarrow{\mathcal{J}}_{D}\right] \cdot \vec{A}(\hat{\Omega}),
\end{aligned}
$$


$c_{S}$ is the density of local moments with spin $S$, and $\vec{A}(\hat{\Omega})=\frac{\hbar c}{4} \cos (\theta) \vec{\nabla} \phi$ is an effective vector potential which depends on the local spin configuration, $\vec{\Omega}$. In Eq. $28, \overrightarrow{\mathcal{J}}_{P}$ and $\overrightarrow{\mathcal{J}}_{D}$ are respectively paramagnetic and diamagnetic contributions to the current density defined by

$$
\begin{aligned}
\overrightarrow{\mathcal{J}}_{P} & \equiv \frac{e \hbar}{2 m i}(\bar{\Phi}(\vec{x}, \tau) \vec{\nabla} \Phi(\vec{x}, \tau)-\vec{\nabla} \Phi(\vec{x}, \tau) \Phi(\vec{x}, \tau)) \\
\overrightarrow{\mathcal{J}}_{D} & \equiv \frac{e}{m c} \bar{\Phi}(\vec{x}, \tau) \Phi(\vec{x}, \tau) \vec{A}(\hat{\Omega}) .
\end{aligned}
$$

The $\overrightarrow{\mathcal{J}}_{P} \cdot \vec{A}$ coupling has the form anticipated by BJZ. To address the magnetic elementary excitation spectrum we formally integrate out the fermion fields $\Phi$ and expand to quadratic order in magnetic fluctuations. The action expressed in terms of only the spin fields is $S_{\text {eff }}(\vec{\Omega})=$ $S_{B}+\operatorname{Tr}\left[\ln \mathcal{G}^{-1}\right]$ with

$$
\begin{array}{r}
\mathcal{G}^{-1}(\theta, \phi)=\partial_{\tau}-\mu-\frac{\hbar^{2}}{2 m}\left(i \vec{\nabla}-\frac{\vec{A}}{2 c}\right)^{2}+ \\
+i \cos (\theta) \partial_{\tau} \phi+\frac{\hbar^{2}}{8 m} \sum_{i, j} \nabla_{i} \Omega_{j} \nabla_{i} \Omega_{j}
\end{array}
$$

Expanding around the $\hat{x}\left(\theta=\frac{\pi}{2} \phi=0\right)$ direction we obtain for the spin-wave action

$$
\operatorname{Tr} \operatorname{Ln}\left[\mathcal{G}^{-1}(\theta, \phi)\right]=\operatorname{Tr} \operatorname{Ln}\left[\mathcal{G}^{-1}\left(\frac{\pi}{2}, 0\right)+\delta \mathcal{G}^{-1}(\vec{\Omega})\right]
$$

To leading order in $\delta \mathcal{G}^{-1}$, the action reads:

$$
\begin{aligned}
& S=S_{B}(n)+\int_{0}^{\beta} \int d \vec{x} \frac{\hbar^{2} n}{8 m} \sum_{i, j} \nabla_{i} \Omega_{j} \nabla_{i} \Omega_{j} \\
& +\frac{2}{e c} \int_{0}^{\beta} \int d \vec{x}\left[\vec{j}+\frac{e n}{m c} \vec{A}(\hat{\Omega})\right] \cdot \vec{A}(\hat{\Omega})
\end{aligned}
$$

where $\vec{j} \equiv \operatorname{Tr}\left[\mathcal{G}\left(0, \frac{\pi}{2}\right) \overrightarrow{\mathcal{J}}_{P}(\vec{x}, \tau)\right]$ is the average current and $n \equiv \operatorname{Tr}\left[\mathcal{G}\left(0, \frac{\pi}{2}\right) \bar{\Phi}(\vec{x}, \tau) \Phi(\vec{x}, \tau)\right]$ is the average density in the collinear ground state. In deriving this expression we allowed the mean-field fermion quasiparticle occupation numbers to assume values consistent with a non-equilibrium current-carrying state.

Equation (31) defines a theory for the collective magnetization of the ferromagnet. The first two terms are the Berry phase of the $d$ spin and a renormalization of the Berry phase due to the spin of the $s$ electrons, similar to that derived by Millis et al. for the double exchange model [27]. The third term describes the energy penalty for non collinear configurations, or spin stiffness. The terms in the second line yield the coupling of the average (paramagnetic and diamagnetic) currents to the collective magnetization.

The semiclassical equations of motion of (31) yield the Landau Lifshitz (LL) equations including the $j_{i} \nabla_{i} \Omega \times \Omega$ term derived by BJZ (equation (5)). In the case of BJZ, the LL equations are derived from a micromagnetic energy functional plus the paramagnetic current term. In our case, the whole functional is derived from the microscopic Hamiltonian. The spin wave expansion for (31) around a classical homogeneous ground state, $\vec{\Omega}_{\mathrm{cl}}=\hat{x}$ is obtained by expanding $\vec{\Omega}=\vec{\Omega}_{\mathrm{cl}}+\delta \vec{\Omega}$ and identifying $\delta \Omega_{y} \simeq \phi, \delta \Omega_{z} \simeq \cos (\theta)$. Dropping terms of order $\delta \Omega^{3}$ and higher, the action (31) becomes:

$$
S_{S W}=\frac{1}{2 \beta V} \sum_{\mathcal{Q}, a b} \delta \Omega_{a}(\mathcal{Q}) \mathcal{K}_{a b}(\mathcal{Q}) \delta \Omega_{b}(-\mathcal{Q})
$$

as in Eq.(15). After analytical continuation, the spin wave kernel 1 , in the $y, z$ representation:

$$
\chi_{\perp}^{-1}=c_{S}\left(\begin{array}{cc}
\rho q^{2} & -i S^{\prime} \omega \\
i S^{\prime} \omega & \rho q^{2}
\end{array}\right)+\frac{\hbar}{e} \vec{j} \cdot \vec{q}\left(\begin{array}{cc}
0 & -i \\
i & 0
\end{array}\right)
$$

where $r \equiv \frac{n}{c_{S}}, \rho \equiv r \frac{\hbar^{2}}{4 m}$, and $S^{\prime}=S+\frac{r}{2}$. The main difference between $s-d$ and Hubbard model result is the appearance here of both local moment and itinerant electron $(r / 2)$ contributions to the Berry phase, which is proportional to the total spin density. Note that since $\vec{A}$ is quadratic in the spin wave variables, the term $\vec{A}^{2}$ in (31) gives no contribution to (32). After diagonalization of Eq. (33) we obtain the retarded propagator for the spin wave variables. The real and imaginary part of the poles of the retarded propagator give the spin wave dispersion and damping, respectively. In this theory, the imaginary part is zero, since the spin flip of quasiparticles is blocked. The real part reads:

$$
\omega=\left[\frac{\hbar^{2} n \vec{q}^{2}}{4 S m}-\frac{\hbar}{2 S e} \vec{j} \cdot \vec{q}\right] \times \frac{1}{c_{S}\left(1+\frac{n}{2 S c_{S}}\right)}
$$

Hence, we see how the spin wave dispersion in this theory has the $\vec{q} \cdot \vec{j}$ term derived by BJZ. Since the system described by the theory is fully polarized, the current and the spin current (polarized along the ground state magnetization direction) are identical. This result is to be compared with Eq. (24), derived with a different method for a different microscopic model. We conclude that spin-wave Doppler shifts due to spin currents are generic, although their quantitative details can depend on the microscopic physics of the ferromagnet.

\section{ENHANCED SPIN-WAVE DAMPING AT FINITE CURRENT}

In Sections III and IV we have shown how the dispersion of spin waves in a metallic ferromagnet is affected by current flow, and we have obtained results compatible with those of BJZ [11]. In this section we address a problem which, to our knowledge, has remained unexplored so far: how does the current flow affect the lifetime of the 
spin waves. In subsection A we analyze the damping of spin waves at zero current. In the subsections B and C we discuss how these results are modified by the presence of a current.

A ferromagnetic resonance (FMR) experiment probes the dynamics of the coherent or $\vec{q}=0$ spin wave mode. The signal linewidth is inversely proportional to the coherent mode lifetime, the time that it takes for a transverse magnetic fluctuation to relax back to zero. Spin waves have a finite lifetime because they are coupled to each other and to other degrees of freedom, including phonons and electronic quasiparticles. In ferromagnetic metals, the quasiparticles are an important part of the dissipative environment of the spin waves 31, 32, 33, 34]. and we can therefore expect that quasiparticle current flow affects the spin wave lifetime to some degree. In order to discuss this effect, it is useful to first develop the theory of quasiparticle spin-wave damping in equilibrium.

\section{Damping at zero current}

The elementary excitation energies for the ferromagnetic phase of the Hubbard model, are specified by the locations of poles in Eq. (17). The damping rate is proportional to the imaginary part of the transverse fluctuation propagator. According to Eq.( 17), the damping of a spin wave with frequency $\omega$ and momentum $\vec{q}$, $\gamma(\vec{q}, \omega)=-2 \operatorname{Im}[\Gamma(\omega, \vec{q})]$ is given by:

$$
\gamma(\vec{q}, \omega)=\frac{2 \pi}{\mathcal{N}} \sum_{\vec{k}}\left[n_{\vec{k}}^{\uparrow}-n_{\vec{k}+\vec{q}}^{\downarrow}\right] \delta\left[\epsilon_{\vec{k}}^{\uparrow}-\epsilon_{\vec{k}+\vec{q}}^{\downarrow}+\omega\right]
$$

In the absence of disorder, this quantity is nonzero when $|\vec{q}|$ is comparable to $k_{F \uparrow}-k_{F \downarrow}$ or when $\omega \simeq \Delta$, the band spin-splitting. Either disorder, which breaks translational symmetry leading to violations of momentum conservation selection rules, or spin-orbit interactions, which cause all quasiparticles to have mixed spin character, will lead to a finite electronic damping rate at characteristic collective motion frequencies. Because this damping is extrinsic, however, its numerical value is usually difficult to estimate. It is often not known whether coupling to electronic quasiparticles, phonons, or other degrees of freedom dominates the damping.

Formally generalizing Eq. (35) to the case with disorder and spin orbit interactions leads to

$$
\gamma(\omega) \propto \sum_{\vec{k}, \vec{k}^{\prime}, \nu, \nu^{\prime}} S_{\nu, \nu^{\prime}}\left(\vec{k}, \vec{k}^{\prime}\right)\left(n_{\vec{k}}^{\nu}-n_{\vec{k}^{\prime}}^{\nu^{\prime}}\right) \delta\left[\epsilon_{\vec{k}}^{\nu}-\epsilon_{\vec{k}^{\prime}}^{\nu^{\prime}}+\omega\right]
$$

where $S_{\nu, \nu^{\prime}}\left(\vec{k}, \vec{k}^{\prime}\right) \equiv\left|\left\langle\vec{k}, \nu\left|S^{(-)}\right| \overrightarrow{k^{\prime}}, \nu^{\prime}\right\rangle\right|^{2}$ is a matrix element between disorder broadened initial and final quasiparticle states, labeled by momentum $\vec{k}$ and band index $\nu$ (but not Bloch states). Averaging out the extrinsic dependence on wavevector labels by letting $S_{\nu, \nu^{\prime}}\left(\vec{k}, \vec{k}^{\prime}\right) \rightarrow$
$S_{\nu, \nu^{\prime}}$ we obtain

$$
\begin{aligned}
\gamma(\omega) & =n^{2} \sum_{\nu, \nu^{\prime}} S_{\nu, \nu^{\prime}} \int d \epsilon \int d \epsilon^{\prime} N_{\nu}(\epsilon) N_{\nu^{\prime}}\left(\epsilon^{\prime}\right) \times \\
& \times\left(n(\epsilon)-n\left(\epsilon^{\prime}\right)\right) \delta\left[\epsilon-\epsilon^{\prime}+\omega\right]
\end{aligned}
$$

where $N_{\nu}(\epsilon)$ is the density of states of the band $\nu$. For $\omega$ of the order of the ferromagnetic resonance frequency, we can expand Eq. (37) to lowest order in $\omega$ :

$$
\gamma(\omega) \simeq \omega\left[n^{2} \sum_{\nu, \nu^{\prime}} S_{\nu, \nu^{\prime}} N_{\nu}\left(\epsilon_{F}\right) N_{\nu^{\prime}}\left(\epsilon_{F}\right)\right]
$$

This result can be considered a microscopic justification of the Gilbert damping law, which states that the damping rate is linearly proportional to the resonance frequency and vanishes at $\omega=0$. The proportionality between frequency and damping rate follows from phase space considerations: the higher the spin wave frequency $\omega$, the larger the number of quasiparticle spin flip processes compatible with energy conservation.

\section{Damping at finite current}

We analyze how a current modifies quasiparticle damping, we again appeal to the picture of magnons as harmonic oscillators coupled to a bath of particle-hole excitations and borrow results from master equation results for oscillators weakly coupled to a bath 25] For magnetization in the ' $\uparrow$ ' direction, magnon creation is accompanied by quasiparticle-spin raising and magnon annihilation is accompanied by quasiparticle-spin lowering. It turns out [25] that only the difference between the rate of quasiparticle up-to-down and quasiparticle down-to-up transitions enters the equation that describes the magnetization evolution. This transition rate difference leads to the same combination of quasiparticle occupation numbers as in Eq. (38), except that the occupation numbers characterize the current-carrying state and are not Fermi factors. For metals we can use the standard approximate form 35. for the quasiparticle distribution function in a current carrying state:

$$
g_{\vec{k}}^{\nu}=n_{\vec{k}}^{\nu}-e \vec{E} \cdot \vec{v}_{\nu}(\vec{k}) \tau_{\nu}\left(\epsilon_{\vec{k}}^{\nu}\right)\left[-\left.\frac{\partial n}{\partial \epsilon}\right|_{\epsilon=\epsilon_{\vec{k}}^{\nu}}\right]
$$

Because of the independent sums over $\vec{k}$ and $\overrightarrow{k^{\prime}}$ in Eq. ( 36 ), and because it is a simple difference of Fermi factors that enters the damping expression, we conclude that the quasiparticle damping correction will vanish to leading order in the spin-dependent drift velocities $v_{D}^{\sigma}$. We reach this conclusion even though the phase space for spin-flip quasiparticle transitions at the spin-wave energy is altered by a factor $\sim 1$ when $\epsilon_{F} \times \frac{v_{D}}{v_{F}} \sim \epsilon_{0}$, where 
$\epsilon_{F}$ is a characteristic quasiparticle energy scale, i.e. the up-to-down and down-to-up transition rates change significantly when this condition is met, but not their difference. To obtain a crude estimate for the current at which this condition is satisfied we use the following data 35$]$ for iron: $n \approx 1.710^{23}$, Fermi velocity $\sim 1.9810^{8} \mathrm{~cm} \mathrm{~s}^{-1}$. The drift velocity corresponding to a current density of $10^{\beta} \mathrm{A}$ $\mathrm{cm}^{-2}$ is $v_{d}=\frac{j}{e n} \simeq 10^{\beta-4} \mathrm{~cm} \mathrm{~s}^{-1}$. The typical energy of a long-wavelength magnon is $\sim 10^{-6} \mathrm{eV}$. Therefore, current densities of the order of $10^{6} \mathrm{~A} \mathrm{~cm}^{-2}$ and larger will substantially change the coupling of spin-waves to their quasiparticle environment. Although this change will influence the spin-wave density-matrix, magnetization fluctuation damping itself will not be altered by this mechanism until much stronger currents are reached.

\section{Two magnon damping}

In the previous subsections we have calculated the damping of the lowest energy spin wave due to its coupling to the reservoir of quasiparticles. In this section we study damping of the coherent rotation mode $(\vec{q}=0$ spin wave) due to its coupling to finite $\vec{q}$ spin waves. This mechanism is known as two magnon scattering and is efficient when the coherent rotation mode is degenerate with finite $\vec{q}$ spin waves 36 , a circumstance that sometimes arises due to magnetostatic interactions. The main point we wish to raise here is that because of the spinwave Doppler shift, precisely this situation arises when the ferromagnet is driven by a current. As in the previous subsection, we assume that some type of disorder lifts momentum conservation. The effective Hamiltonian for the spin waves reads:

$$
H=\omega_{0} b_{0}^{\dagger} b_{0}+\sum_{\vec{q} \neq 0} \omega(\vec{q}) b_{\vec{q}}^{\dagger} b_{\vec{q}}+b_{0}^{\dagger} \sum_{\vec{q} \neq 0} \frac{g_{\vec{q}}}{\sqrt{V}} b_{\vec{q}}+\text { h.c. }
$$

where $b_{\vec{q}}$ is the annihilation operator for the spin wave with momentum $\vec{q}$ and $g_{\vec{q}}$ is some unspecified matrix element accounting for disorder induced elastic scattering of the spin waves. Equation (40) is the well Hamiltonian known for a damped harmonic oscillator and can be solved exactly. The damping rate for the $\vec{q}=0$ spin wave reads:

$$
\gamma(\overrightarrow{\mathcal{J}})=\frac{2 \pi}{\hbar} \int \frac{d \vec{q}}{(2 \pi)^{3}}\left|g_{\vec{q}}\right|^{2} \delta\left(\omega_{0}-\omega_{\vec{q}}\right)
$$

Now we use $\omega_{0}-\omega_{\vec{q}}=\rho q^{2}-a \vec{q} \cdot \overrightarrow{\mathcal{J}}$. After a straightforward calculation we obtain:

$$
\gamma(\overrightarrow{\mathcal{J}})=\frac{g^{2}}{4 \pi} \frac{a|\overrightarrow{\mathcal{J}}|}{\rho^{2}}
$$

where we have approximated $g_{\vec{q}} \simeq g$. Hence, in the presence of elastic spin wave scattering, renormalization of the spin wave spectrum due to the current will enhance the damping of the lowest spin wave mode. Unlike the Gilbert model, the damping rate given by equation (42) is independent of $\omega_{0}$, implying that the dimensionless Gilbert damping coefficient would decline with external field if this mechanism were dominant.

\section{SPIN-WAVE DOPPLER SHIFT AS A SPIN-TORQUE EFFECT}

In this section we explain how the influence of an uniform current on magnetization dynamics can be understood as a special case of a spin-torque effect [2, 3]. The latter takes place when a spin current coming from a magnet spin polarized along $\vec{M}_{1}$ enters in a magnet spin polarized along $\vec{M}_{2}$. In this circumstance there is an imbalance between the incoming and the outgoing transverse component (with respect to $\vec{M}_{2}$ ) of the spin currents in magnet 2. Because of spin conservation (resulting from the rotational invariance of the system), the imbalance in the spin flux across the boundaries of magnet 2 must be compensated by a change of the magnetization of that magnet, which is described by a new term in the Landau Lifshitz equation [2, 3]. The microscopic origin of the spin current imbalance can be understood as a destructive interference effect, originated by the fact that the steady state spin current is a sum over stationary states with broad distribution in momentum space [2]. Alternatively, it is possible to understand the spin current flux imbalance as a destructive interference in the time domain. At a given instant of time, the outgoing current-carrying quasiparticles have elapsed a different amount of time in magnet 2 . This broadening in the interaction time distribution results in a broadening of the spin precession angle 37]. The average over that distribution results in a vanishing transverse spin component of the outgoing flux.

The above argument, connecting spin flux imbalance and spin-torque, applies to a system in which the inhomogeneous magnetization is described by piecewise constant function. It is our contention that the spin wave Doppler shift can be understood by applying the same argument to the case of smoothly varying magnetization. We consider again a magnet with charge current $\vec{j}$, and spin current $\overrightarrow{\mathcal{J}}$. We assume that current flows in the $\hat{x}$ direction and, importantly, that the spin current is locally parallel to the magnetization orientation $\overrightarrow{\mathcal{J}}(x)=j_{s} \hat{\Omega}(x)$. It can be shown that this is the case in a wide range of situations.

The spin density reads $\vec{S}(x)=S_{0} \hat{\Omega}(x)$ where $S_{0}$ is the average spin polarization. We focus on the slab centered at $\mathrm{x}$ and bounded by $x-d x$ and $x+d x$. Spins are injected into the slab at the rate $j_{s} \hat{\Omega}(x-d x)$ and leave at the rate $j_{s} \hat{\Omega}(x+d x)$. The resulting spin current imbalance is $2 d x j_{s} \partial_{x} \hat{\Omega}$. Therefore, there must be a spin transfer to 
the local magnetization:

$$
\left.\frac{d \vec{S}(x)}{d t}\right|_{S T}=j_{s} \partial_{x} \hat{\Omega}
$$

Now, using $|\hat{\Omega}|^{2}=1$ at every point of the space we obtain:

$$
\left.\frac{d \vec{S}(x)}{d t}\right|_{S T}=j_{s} \hat{\Omega}(x) \times\left(\partial_{x} \hat{\Omega}(x) \times \hat{\Omega}(x)\right)
$$

which is exactly the same result obtained in 11 . Including this term in the Landau Lifshitz equation and solving for small perturbations around the homogeneous ground state (spin waves) results into the spin wave Doppler shift discussed in previous sections. In conclusion, this argument demonstrates that the spin-wave Doppler shift and spin transfer torques are different limits of the same physical phenomena, the transfer of angular momentum from the quasiparticles to the collective magnetization whenever the latter is not spatially uniform.

\section{DISCUSSION AND CONCLUSIONS}

The effect of high current densities on the magnetization dynamics of ferromagnetic metals have been explored experimentally in several configurations. In point contact experiments, a large current density is injected from a normal metallic contact into a ferromagnetic multilayer [4, 5] or single layer 8]. When a large flow of electrons ( current density $\mathrm{j} \simeq 10^{8} \mathrm{~A} \mathrm{~cm}^{-2}$ ) enters into the ferromagnetic multilayer, the resistivity presents an abrupt increase which has been related to the coherent precession of spin waves 4] and/or phonons [5]. The fact that Ji and Chien [8] report similar results when the current is injected into a a single ferromagnetic layer demonstrates that interlayer coupling is not essential for the anomalies observed in transport. It must be noted that when the current flow is such that the electrons go from the ferromagnetic layer(s) toward the point contact, no anomaly is observed. Similar transport anomalies at currents densities higher than those of current induced magnetization switching are observed by a number of different groups [7] in a system of two adjacent ferromagnetic nanopillars. In this system a large current density flows from one ferromagnet to the other.

The fact that the current densities at which the anomalous behavior takes place is of the same order of magnitude than the current at which the spin wave Doppler shift makes the collinear state unstable might lead to suggest a connection between the two. However, the experiments in the point contact geometry show that the transport anomalies only occur for one direction of the current, something which seems at odds with the spin wave Doppler shift instability.
In summary, the focus of this paper is on the effect of the current in the spin wave dynamics of a bulk ferromagnetic metal. We have addressed two types of effects: the change in the spin wave dispersion and change in the spin wave damping. These quantities are given, at a formal level, by the spin wave propagator. The central idea is that the spin wave propagator is a functional of the quasiparticle occupation function. In the presence of the current the occupation function changes, affecting both the dispersion and the damping of the spin waves. Throughout the paper we have assumed that the functional relation between the quasiparticle occupation function and the spin wave propagator remains the same when the system is out of equilibrium. In that sense, the above derivations are heuristic. Our main conclusions are: i) A current $\vec{j}$ flowing through a metallic ferromagnet results 30$]$ in a spin current $\overrightarrow{\mathcal{J}}$ which modifies its spin wave spectrum by an amount proportional to $\vec{q} \cdot \overrightarrow{\mathcal{J}}$. ii) This modification, which was derived by BJZ for a fully polarized s-d model, occurs as well in a non fully polarized Hubbard model, in which the $d$ electrons are itinerant and, according to the arguments of section II, in typical real-world ferromagnets. iii) In the presence of elastic two magnon scattering, the spin wave Doppler shift leads as well to a broadening of the lowest spin wave mode (Eq. 42), which is proportional to the spin current. iv) Both the spin-wave Doppler shift in spatially homogeneous ferromagnets and the spin torque effect in inhomogenoeus structures 2, 3] are a consequence of the spin transfer from the quasiparticles to the collective magnetization when the latter is spatially inhomogeneous.

We acknowledge fruitful discussions with M. Abolfath and M. Tsoi. Work at the University of Texas was supported by the Welch Foundation and by the National Science Foundation under grants DMR-0210383 and DMR0115947. Work at University of Alicante supported by Ramón y Cajal Program, Ministerio de Ciencia y Tecnología.

[1] Spin Dependent Transport in Magnetic Nanostructures, Ed. by S. Maekawa and T. Shinjo, (Taylor and Francis, 2002).

[2] J.C. Slonczewski, J. Mag. Mat. Mag. 159, L1 (1996).

[3] L. Berger, Phys. Rev. B54, 9353 (1996).

[4] M. Tsoi et al., Phys. Rev. Lett. 80, 4281 (1998); M. Tsoi et al., Nature 406, 46 (2000);

[5] M. Tsoi et al., Phys. Rev. Lett. 89, 246803 (2002).

[6] J.Z. Sun, J. Magn. Mag. Mater. 202, 157 (1999).

[7] E. B. Myers et al., Science 285, 867 (1999) J.A. Katine et al., Phys. Rev. Lett. 84, 4212 (2000); E.B. Myers, et al., Phys. Rev. Lett. 89, 196801, (2002); S.I. Kiselev em et al., preprint cond-mat/0306259 (2003). W.H. Rippard, M.R. Pufall, and T.J. Silva, Appl. Phys. Lett. 82, 12601262 (2003). F. B. Mancoff, et al. Appl. Phys. Lett. 83, 1596 (2003). 
[8] Y. Ji and C.L. Chien and M. D. Stiles, Phys. Rev. Lett. 90, 106601 (2003).

[9] S. Urazhdin et al., Phys. Rev. Lett. 91, 146803 (2003)

[10] J. Z. Sun, Phys. Rev. B 62, 570 (2000 ); A. Brataas, Y. V. Nazarov, and G.E.W. Bauer, Phys. Rev. Lett. 84, 2481 (2000); X. Waintal and P.W. Brouwer, Phys. Rev. B 63, 220407 (2001); C. Heide, Phys. Rev. B 65, 054401 (2002); M. Stiles and A. Zangwill, Phys. Rev. B 65, 014407 (2002); J.-E. Wegrowe, Appl. Phys. Lett. 80, 3775 (2002); S. Zhang, P.M. Levy, and A. Fert, Phys. Rev. Lett. 88, 236601 (2002); G.E.W. Bauer, Y. Tserkovnyak, D. Huertas-Hernando, and A. Brataas, Phys. Rev. B 67, 094421, (2003); M.L. Polianski and P.W. Brouwer, preprint cond-mat/0304069 (2003); A. Shapiro, P. M. Levy, and S. Zhang Phys. Rev. B 67, 104430 (2003); A. Fert et al., preprint cond-mat/0310737 (2003).

[11] Y.B. Bazaliy, B. A. Jones and S.-C. Zhang, Phys. Rev. B57, R3213 (1998)

[12] More precisely, $\overrightarrow{\mathcal{J}} \equiv \frac{e}{\hbar \mathcal{N}} \sum_{\vec{k}} \frac{\partial \epsilon(\vec{k})}{\partial \vec{k}}\left[n_{\vec{k}}^{\uparrow}-n_{\vec{k}}^{\downarrow}\right]$, where $\mathcal{N}$ is the number of sites in the lattice, and $\uparrow$ and $\downarrow$ are defined in the axis of the average magnetization.

[13] C. Herring and C. Kittel, Phys. Rev. 81, 869 (1951).

[14] E.P. Wolfarth, Rev. Mod. Phys. 25, 211 (1953); D.A. Papaconstantopoulos, Handbook of the Band Structure of Elemental Solids (Plenum Press, New York, 1986).

[15] R. E. Prange and V. Korenman, Phys. Rev. B 19, 4691 (1979)

[16] T. Moriya, Spin Fluctuations in Itinerant Electron Magnetism, (Springer-Verlag, Berlin, 1985)

[17] J. Hubbard, Phys. Rev. B19, 2626 (1979)

[18] H.J. Schulz, Phys. Rev. Lett65, 2462 (1990)

[19] Sections 3.3 and 3.4 in E. Fradkin, Field Theories of Condensed Matter Systems, (Addison Wesley, Reading, MA, 1991)
[20] S. Doniach and E. H. Sondheimer, Green's Functions for Solid State Physicists, W. A. Benjamin, Inc (1974)

[21] R.L. Stratonovitch, Dok. Akad. Nauk. SSSR 115, 1097 (1957); [Sov. Phys. Dokl. 2, 416 (1958)]. J. Hubbard, Phys. Rev. Lett. 3, 77 (1959)

[22] J.W. Negele and H. Orland, Quantum Many-Particle Systems, Addison Wesley, (1988)

[23] Assa Auerbach, Interacting Electrons and Quantum Magnetism, Springer-Verlag, 1994.

[24] R. Skomski and J. M. D. Coey, Permanent Magnetism (Institute of Physics, Bristol, 1999).

[25] Claude Cohen-Tannoudji, Gilbert Grynberg, Jacques Dupont-Roc Atom-Photon Interactions: Basic Processes and Applications, (Wiley-Interscience, New York,1998)

[26] V. Korenman, J. L. Murray, and R. E. Prange Phys. Rev. B 16, 4032 (1977)

[27] A. J. Millis, P.B. Littlewood and B. I. Shraiman, Phys. Rev. Lett74, 5144 (1995)

[28] P. M. Levy and S. Zhang, Phys. Rev. Lett. 79 , 5110 (1997)

[29] G. Tatara and H. Fukuyama, Phys. Rev. Lett. 78, 3773 (1997)

[30] A. Fert, J. Phys. C. 2, 1784 (1969) and references therein.

[31] V. Kambersky, Canadian Journal of Physics, 482906 (1970)

[32] V. Kambersky and C. E. Patton, Phys. Rev. B 11, 2668 (1975)

[33] V. Korenman, J. L. Murray, and R. E. Prange Phys. Rev. B 16, 4048-4057 (1977)

[34] L. Berger, J. Phys. Chem. Solids 38, 1321 (1977)

[35] N. W. Ashcroft and N. D. Mermin, Solid State Physics, W.B. Saunders Company (1976)

[36] R. Arias and D. Mills, Phys. Rev. B 60, 7395 (1999)

[37] J. Fernandez-Rossier et al., cond-mat/0304492 (2003) 